\title{
Three Component Doppler Global Velocimeter Measurements of the Flow Above A Delta Wing
}

James F. Meyers

Joseph W. Lee

NASA Langley Research Center

Hampton, Virginia

and

Angelo A. Covone ViGYAN, Inc.

Hampton, Virginia

Sixth International Symposium on Applications of Laser Techniques to Fluid Mechanics

July 20-23, 1992

Lisbon, Portugal 



\title{
Three Component Doppler Global Velocimeter Measurements of the Flow Above A Delta Wing
}

\author{
by \\ James F. Meyers \\ Joseph W. Lee \\ NASA - Langley Research Center \\ Hampton, Virginia 23665 \\ and \\ Angelo A. Cavone \\ ViGYAN, Inc. \\ Hampton, Virginia 23666
}

\begin{abstract}
A new measurement technique is being developed by NASA to measure off-surface flow fields. This method, Doppler global velocimetry, will allow quantification of complex three-dimensional flow fields at video camera rates. The entire flow field structure within a selected plane is measured simultaneously rather than by scanned, point-by-point measurements using conventional laser velocimetry. To assess the capability of this new technique, three-component velocity measurements of the vortical flow field above a thin 75-degree delta wing were made in the NASA Langley Basic Aerodynamics Research Tunnel. Measurements were made of the flow field at the 70 -percent chord location at angles-of-attack of 20.5- and 40.0-degrees to investigate unburst and burst vortices. For comparison, previous fringe-type laser velocimeter measurements of the flow field at the same conditions are included.
\end{abstract}

\section{Nomenclature}

ALF Absorption line filter, Iodine vapor

c Speed of light, $\mathrm{m} / \mathrm{sec}$

$\hat{i} \quad$ Laser beam propagation direction 
ô Collected scattered light direction

$\mathrm{V}$ Velocity of a particle passing through the laser beam, $\mathrm{m} / \mathrm{sec}$

$X \quad$ Cross tunnel coordinate, $m$

$Y \quad$ Vertical coordinate, $\mathrm{m}$

$Z \quad$ Streamwise coordinate, $m$

$\Delta v \quad$ Doppler shift frequency, $\mathrm{Hz}$

$v \quad$ Laser output frequency, $\mathrm{Hz}$

$\theta \quad$ Angle between the laser propagation direction and the collected scattered light, deg

\section{Introduction}

Of the many fundamental flow structures comprising aircraft fluid dynamics, vortical flows are receiving increased attention. Vortices, if properly utilized, can provide increased lift to enhance maneuverability of modern high-performance aircraft. Devices such as leading edge extensions and extended forebodies have produced vortical flows which have increased the performance of aircraft such as the F-15 and the F/A-18. These devices were developed using the trial-and-error method of aircraft design using force and moment response of wind tunnel models to determine increases in performance. This method provides little, if any, information on dynamic loading on aircraft structures, nor off-body flow field information to correlate flow dynamics with force and moment data. Even if off-body flow field investigations were conducted, classic approaches using probes would change the flow since vortices will avoid obstacles and move around the probes. The only known nonintrusive measurements of the flow about a highperformance configuration were obtained years after the aircraft was operational, reference 1 . This investigation, using fringe-type laser velocimetry to measure the off-body flow above a YF-17 at high anglesof-attack, found a complex flow field containing burst vortices and flow separation over 60 percent of the wing. The trajectory of the burst vortex intersected the vertical stabilizer at mid span imparting large dynamic forces on the stabilizers, which may have caused the fatigue damage found in F/A-18's. 
Although laser velocimetry measures the flow without disturbance, this point measurement technique needs a great deal of time to map a flow field. This requires the flow to be stationary during acquisition of the velocity data. Present code predictions and laser velocimetry measurements of vortical flows appear to support the assumption that these flows are stationary. However, standard deviations of the measurement ensembles, reference 1, indicate large departures of velocity from the mean, especially in burst vortices. The dynamics of these variations have a direct bearing on the loading of aircraft structures. If the velocity variations are spatially random, structural loading will likewise be random, dispersing the impact energy. However, if the variations are spatially organized, dynamic loading may cause structural fatigue. Global measurement techniques represent the only means of determining the instantaneous spatial characteristics of the velocity field.

Recent developments in global velocimetry have produced three possible candidates: particle image velocimetry, reference 2; Doppler pictures obtained with a Michelson interferometer, reference 3; and, Doppler global velocimetry, reference 4. Particle image velocimetry uses a double pulse laser to illuminate a measurement plane within the flow. Scattered light from the particles present within the plane is collected and imaged on photographic film. The double exposures recorded on film are interrogated to determine the distance traveled within the plane by each recorded particle during the time between pulses. The limitation of two component measurements causes particle image velocimetry to be unsuited for the investigation of the three dimensional vortical flow field. Doppler pictures are constructed using a Michelson interferometer to produce fringe patterns indicating lines of constant phase shift of the Doppler shifted light scattered by particles passing through a laser light sheet. These spatial fringe patterns outline the velocity contours within the imaged measurement plane. Although three-component Doppler pictures can be obtained, the Michelson interferometer is a complicated optical configuration producing fringe images that are difficult to process. Doppler global velocimetry also uses a laser light sheet to illuminate a particle field, and like the Doppler picture method, the technique measures the frequency shift of the scattered light. Instead of interferometry, the technique uses the optical transfer function of an absorption line in Iodine vapor as an optical frequency discriminator. Viewing the light sheet through an Iodine vapor cell with a video camera produces images whose intensity patterns map the velocity flow field. Although the investigation of the vortical flow above a delta wing was chosen as the Doppler global velocimetry proof-of-concept experiment, the technique's characteristics would also make it the prime instrumentation candidate to investigate the aerodynamics of this flow. 


\section{The Doppler Global Velocimeter}

The fundamental physics utilized in the Doppler Global Velocimeter, DGV, is the frequency shift imposed on light scattered from objects passing through a laser beam. This principle was first exploited by Yeh and Cummins in 1964, reference 5, to develop the reference-beam, laser Doppler velocimeter, LDV. As depicted in figure 1, scattered light collected by a detector located along the direction $\hat{o}$, from particles passing through a laser beam propagating in direction $\hat{1}$, is Doppler shifted based on a velocity in the direction ( $\hat{o}-\hat{1})$. This relationship is expressed by:

$$
\Delta v=\frac{v_{o}(\hat{\mathrm{o}}-\hat{\mathrm{1}}) \bullet \mathrm{V}}{\mathrm{c}}
$$

where $\Delta v$ is the Doppler shifted frequency, $v$ is the laser frequency, $V$ is the particle velocity, and $c$ is the speed of light. The angular dependence of the Doppler shift magnitude on system geometry, as indicated by the dot product in equation (1), presents the possibility of three-component velocity measurements by using various laser propagation directions and/or locations for the receiver optical system.

Although equation (1) indicates that the Doppler shift principle provides the necessary measurable parameter, the task is to develop a method and the instrumentation to measure that parameter. Yeh and Cummins mixed a portion of the scattered light with unshifted laser light on a photocathode surface to obtain heterodyne detection, thus isolating the Doppler frequency, whereas Self, reference 6, used a Fabry-Perot interferometer to measure the shifted light frequency directly. These techniques have characteristically poor signal-to-noise ratios, and complicated, alignment sensitive, optical systems. A more robust system using a Michelson interferometer was constructed by Smeets, reference 7 . As the fringes produced by the interferometer pass over a spatially filtered photomultiplier, an electronic signal containing the Doppler frequency is output. While this technique increases the signal-to-noise ratio, it still requires a complicated, alignment sensitive, optical system. Komine, et al, reference 8, realized that the optical transfer function of an absorption line in Iodine vapor could be used as an optical frequency discriminator. An Argon ion laser operating in single-line mode at $514.5 \mathrm{~nm}$ is tuned by adjusting the intercavity etalon to an optical frequency corresponding to a point midway along the edge of an Iodine absorption line, figure 2 . Collected scattered light from a stationary object or cloud of particles will be attenuated by 50 percent as it passes through the Iodine vapor. If the object or particle cloud is moving, the attenuation through the vapor will increase (or decrease, depending on the direction of 
movement) by an amount proportional to the Doppler shift. This simple idea has resulted in a velocity measuring technique that has the potential for good signal-to-noise ratios, permitting accurate measurements, while keeping the optical system simple. In practice, particle size distribution and number density, and the laser beam intensity profile also influence the magnitude of the scattered light. Therefore, a second detector is used to measure a portion of the collected light, thus serving as a reference of absolute scattered light intensity. Normalization of the signal detector output, by the reference detector output, results in a signal whose intensity is directly related to the Doppler shift of the scattered light.

While the discussion has been limited to point measurement techniques, the Michelson interferometer and the Iodine cell methods can be expanded to global measurement systems. The expansion consists of fanning the laser beam into a light sheet and replacing the detectors with CCD video cameras. The Michelson interferometer technique, as implemented by Seiler and Srulijes, reference 3, produces images with fringe patterns indicating lines of constant phase shift outlining contours of constant velocity. Their work in supersonic flow indicates the potential of the interferometer to produce Doppler pictures in high-speed, short run time facilities. The Iodine cell technique or Doppler global velocimetry, as implemented by Meyers and Komine, reference 4, produces images whose intensity at any pixel is a measure of the average velocity of the flow passing through the light sheet viewed by that pixel. Although these two techniques have many similarities, the Doppler global velocimeter is the method of choice since it has a simpler optical system and its measurement images are easier to interpret.

The DGV was configured as illustrated in figure 3 for the measurement of the vortical flow above a 75 -degree delta wing in the NASA Langley Basic Aerodynamic Research Tunnel, BART. Three component measurements were obtained by routing the input laser beam through each side window and the ceiling of the test section in sequence. This placed the fixed receiver optical system, shown in figure 3, in forward scatter, back scatter, and side scatter modes, respectively. A portion of the scattered light was collected by a $35 \mathrm{~mm}$ camera lens with a focal length variable from $35 \mathrm{~mm}$ to $105 \mathrm{~mm}$. A fast, spherical transfer lens was placed behind the collecting len s to collimate the light, and pass it to a beam splitter. The beam splitter reflected a portion of the light along a path to the reference camera, while passing the remaining light through the Iodine vapor cell to the signal camera.

The output images from the two CCD video cameras were acquired by a specially designed digital image processor. The processor provided the 
necessary synchronization signals to the two cameras insuring temporal coincidence of the integration of scattered light from the particles passing through the light sheet during each camera frame. The synchronized images were simultaneously recorded by parallel frame grabbers using eight-bit video analog-to-digital converters. The resulting digitized signal image was normalized by the digitized reference image using a table look-up method, reference 9 . The original signal and reference images along with the normalized signal image were passed to the data acquisition microcomputer for final storage. Real-time signal processing was performed using analog divider circuitry in a separate signal processing system, reference 9. Pseudo colors were added to the output video signal from the analog processor using a standard video frame grabber placed in the data acquisition microcomputer. The video signal was then recorded on an optical video disk.

While this straight-forward signal processing technique appears to provide the necessary normalized image, it assumes that the pixel-bypixel alignment between the two cameras is perfect. The experiences during testing in BART and the post-testing analysis of the data indicated that perfect alignment could not be obtained. Detailed laboratory investigations of the receiver optical system following the wind tunnel investigation found several problems preventing the exact overlay of the two images. These problems range from mismatched depths of field, and optical distortions caused by lens aberrations, imperfections in the beamsplitters and mirrors, and the Iodine vapor cell to the nonuniformity of the pixel distribution and element orientation in the cameras. Although these obstacles may not be insurmountable, image processing techniques were investigated as a possible mechanism to improve image alignment, reference 10 .

The method implemented in software was based on determining the set of linear equations needed to move the centroid position of each grid point on a calibration card from its imaged position to its ideal position. This procedure produced image alignment to subpixel accuracy over the entire normalized image. The acquired wind tunnel data were processed again using this technique to overlay the images prior to normalization. Variations in sensitivity among the pixels in each CCD camera were also removed by using flat field calibration procedures.

\section{Flow Field Investigation Above a 75-degree Delta Wing}

The investigation of the vortical flow above a 75-degree delta wing was chosen as the experimental demonstration of the Doppler global 
velocimeter. The intent of this work was to obtain flow velocity distributions that would be comparable with results from a previous three-component, fringe-type laser velocimeter investigation, reference 11, also conducted in the BART. A further goal of the investigation was to demonstrate the ability of near real-time global measurements to provide further insight in to flow field phenomenon than possible with scanned point measurement techniques.

The Basic Aerodynamics Research Tunnel, reference 12, was an open return tunnel with a test section $0.71 \mathrm{~m}$ high, $1.02 \mathrm{~m}$ wide and $3.07 \mathrm{~m}$ long. A maximum velocity of $67 \mathrm{~m} / \mathrm{sec}$ could be obtained in the test section with a test Reynolds number per meter of 0.43 million. The airflow entering the test section was conditioned by a honeycomb structure, four antiturbulence screens, and an 11:1 contraction ratio. The freestream turbulence intensity was less than 0.08 percent for all flow conditions. The propylene glycol vaporization/condensation generator developed for vapor screen flow visualization was used as the source of particles for the experiment. The particles were injected upstream of the hon eycomb structure and have a size distribution which peaks at $0.7 \mu \mathrm{m}$ with a skewed distribution to a maximum of $10 \mu \mathrm{m}$, reference 11 .

The stainless steel 75 -degree delta wing, $0.57 \mathrm{~m}$ in length, with sharp leading edges was placed in the tunnel at an angle of attack of 20.5 degrees. The tunnel dynamic pressure was set to $402 \mathrm{~N} / \mathrm{m}^{2}$ which yields a freestream velocity of $40 \mathrm{~m} / \mathrm{sec}$. An Argon-ion laser operating in $\mathrm{TEM}_{\text {oo }}$ mode with an etalon to maintain a single longitudinal mode at $514.5 \mathrm{~nm}$ was used as the light source. The output beam was directed through one of three cylindrical lenses to form a light sheet placed perpendicular to the tunnel centerline at the 70 -percent chord location on the model. Each of the three cylindrical lenses was placed along an optical path to bring the light sheet into the test section from each side and the top, respectively, resulting in optical configurations that would measure three velocity components. The receiver optical system was located 53 degrees from the streamwise (tunnel centerline) direction in the horizontal plane, figure 3. A photograph of the receiver optical system viewing particles in the vortical flow passing through the light sheet above the delta wing is shown in figure 4 .

The LV measurements of the vortical flow above the delta wing at an angle-of-attack of 20.5 degrees, is shown in figure 5 . The gray scale represents contours of streamwise velocity and the arrows represent the velocity vector of the circular flow within the plane perpendicular to the streamwise direction. Note that the streamwise velocity was accelerated to twice the freestream value at the vortex core. The circular flow was compressed by the wing and accelerated to 1.5 times 
the freestream value as the flow expanded outward below the core. Of the three measurement directions established by the DGV geometry, the direction that best illustrated the circular flow characteristics of the vortex was the nearly cross flow component, 71.5 degrees from the streamwise direction in the horizontal plane, figure 6 . Resolving the three-component LV data to obtain the velocity field along this direction yielded the contour map shown in figure 7 . An average of 30 frames of DGV data (the equivalent of one second of acquisition) acquired by the digital signal processor of the flow field along this component is shown in figure 8 . The characteristic velocity pattern for a vortex flow in the cross flow direction is clearly seen in the LV data and the left vortex in the DGV data. However, the right vortex in the DGV data more closely matches the expected velocity distribution for a solid body of revolution. While the right vortex has not burst, as indicated by the other component measurements, it may represent the transition to a burst condition since increasing the angle-of-attack of the model results in the right vortex bursting first. This asymmetric bursting has been traced to a yaw of 0.2 degrees of the model caused by the mounting hardware.

The vertical component, illustrated in figure 9, is aligned along a direction 53 degrees from streamwise and 45 degrees above the horizontal plane. Resolving the LV data along this direction results in the velocity map shown in figure 10 . Again 30 frames of DGV data were averaged to yield the velocity mapping shown in figure 11 . The third and streamwise component is illustrated in figure 12. The resolved LV data and the averaged DGV data are shown in figures 13 and 14, respectively.

The delta wing was adjusted to 40.0 degrees angle-of-attack to investigate a flow containing burst vortices. The three component measurements obtained with the LV are shown in figure 15. Note that the streamwise velocity has reversed direction within the core region. The flow within the plane normal to the streamwise direction still contains a strong circulation flow. This conflicts with laser light sheet visualization which suggests a chaotic flow without structure. Resolving the LV data to obtain the velocity components measured by the DGV yields the velocity contours in figures 16,18 , and 20 with the corresponding DGV measurements in figures 17,19 , and 21 . The figures indicate that both instruments obtained similar average measurements of the burst vortices. The large amplitudes of the normalized standard deviations obtained with the LV presented in reference 11 indicate large velocity deviations from the mean. Whether these deviations were spatially structured or random with time cannot be determined from the LV data. However, differences between individual frames of DGV data indicate that these variations are spatially structured and to some extent, repeatable. That is, similar spatial distributions of velocity 
occur at different times, but between occurrences, other structures are observed. Also, at times the burst point on the left moves through the light sheet plane yielding an image of a formed vortex on the left and a burst vortex on the right, figure 22 .

Although the present experiment was designed to match the LV investigation as closely as possible, differences still existed. An assessment of the unburst vortices indicates good agreement in the rotating components, but questionable agreement in the streamwise direction. The nonoverlapping vortex centers between the LV and DGV data were traced following the test to a 0.5 -degree roll of the model during the LV tests. Conversely the measurements of the burst vortices yielded good agreement in the streamwise direction, but questionable agreement in the rotating components. Most likely the spread of particles in the burst vortex flow along with the lower scattering efficiency of side scatter and backscatter configurations caused a decrease in scattered light, especially in the left vortex, reducing the amount of reliable data available for processing. The question of whether the remaining differences can be traced to the comparison of the eight hours of data acquisition required by the LV to measure a plane of data, to effectively one second of acquisition using an experimental instrumentation system several years apart, still exists. Or, are these differences actually aerodynamic in nature? Are vortical flows, which appear to be extremely stable, instead very sensitive to the incoming flow and the relation of the model to it? Do burst vortices, thought to be chaotic, have a time varying structure that is spatially repeatable? The irony in these questions is that the Doppler global velocimeter may be the only measurement technique that can provide their answers.

\section{Summary}

A new measurement technique, Doppler global velocimetry, has been described, along with results from an experimental investigation of the vortical flow field above a delta wing. The results of this investigation indicate that this robust technique is capable of describing the entire three-component velocity flow field simultaneously within a measurement plane in real time. Aerodynamically, these results have indicated the possible presence of a transition region between burst and unburst portions of the vortex flow. Als o, evidence of spatial correlation of velocity within the burst vortex has been found. Further detailed investigations of this flow using the Doppler global velocimeter to answer the questions raised by the present investigation will be conducted when the capabilities and accuracies of the technique have been further defined. 


\section{References}

1. Sellers, W. L., III; Meyers, J. F.; and Hepner, T. E.: LDV Surveys over a Fighter Model at Moderate to High Angles of Attack, SAE 1988 Aerospace Technology Conference \& Exposition, Anaheim, CA, October 3-6, 1988.

2. Adrian, R. J.: Scattering Particle Characteristics and Their Effect on Pulsed Laser Measurements of Fluid Flow: Speckle Velocimetry vs. Particle Image Velocimetry, Applied Optics, 23, pp. 1690-1691, 1984.

3. Seiler, F.; and Srulijes, J.: Doppler-Pictures of Velocity Fields, An Application to Fluid Mechanics, Proceedings of the Third International Symposium on Applications of Laser Anemometry to Fluid Mechanics, Lisbon, Portugal, paper 19.1, July 7-9, 1986.

4. Meyers, J. F.; and Komine, H.: Doppler Global Velocimetry - A New Way to Look at Velocity, ASME 4th International Conference on Laser Anemometry, Advances and Applications, Cleveland, OH, August 5-9, 1991.

5. Yeh, Y.; and Cummins, H. Z.: Localized Fluid Flow Measurements with a He-Ne Laser Spectrometer, Applied Physics Letters, vol. 4, no. 10, pp. 176-178, May 1964.

6. Self, S. A.: Laser Doppler Anemometer for Boundary Layer Measurements in High Velocity, High Temperature MHD Channel Flows, Proceedings of the Second International Workshop on Laser Velocimetry, Purdue University, vol. II, pp. 44-67, March 2729,1974 .

7. Smeets, G.: Laser Doppler Velocimetry with a Michelson Spectrometer, Laser Anemometry in Fluid Mechanics, Selected Papers from the First International Symposium on Applications of Laser-Doppler Anemometry to Fluid Mechanics, editors: R. J. Adrian, D. F. G. Durao, F. Durst, H. Mishina and J. H. Whitelaw, Ladoan-Instituto Superior Tecnico, pp. 355-370, 1984

8. Komine, H.; Brosnan, S. J.; Litton, A. B.; and Stappaerts, E. A.: Real Time, Doppler Global Velocimetry, AIAA 29th Aerospace Sciences Meeting, Reno, NV, paper no. AIAA-91-0337, January 7$10,1991$.

9. Meyers, J. F.; Lee, J. W.; and Cavone, A. A.: Signal Processing Schemes for Doppler Global Velocimetry, IEEE - 14th 
International Congress on Instrumentation in Aerospace Simulation Facilities, Rockville, MD, October 27-31, 1991.

10. Meyers, J. F.: Doppler Global Velocimetry - The Next Generation? AIAA 17th Aerospace Ground Testing Conference, Nashville, TN, paper no. AIAA-92-3897, July 6-8, 1992.

11. Meyers, J. F.; and Hepner, T. E.: Measurement of Leading Edge Vortices from a Delta Wing Using a Three Component Laser Velocimeter, AIAA 15th Aerodynamic Testing Conference, San Diego, CA, paper no. AIAA-88-2024, May 18-20, 1988.

12. Sellers, W. L., III; and Kjelgaard, S. O.: The Basic Aerodynamics Research Tunnel - A Facility Dedicated to Code Validation, AIAA 15th Aerodynamic Testing Conference, San Diego, CA, paper no. AIAA-88-1997, May 18-20, 1988.

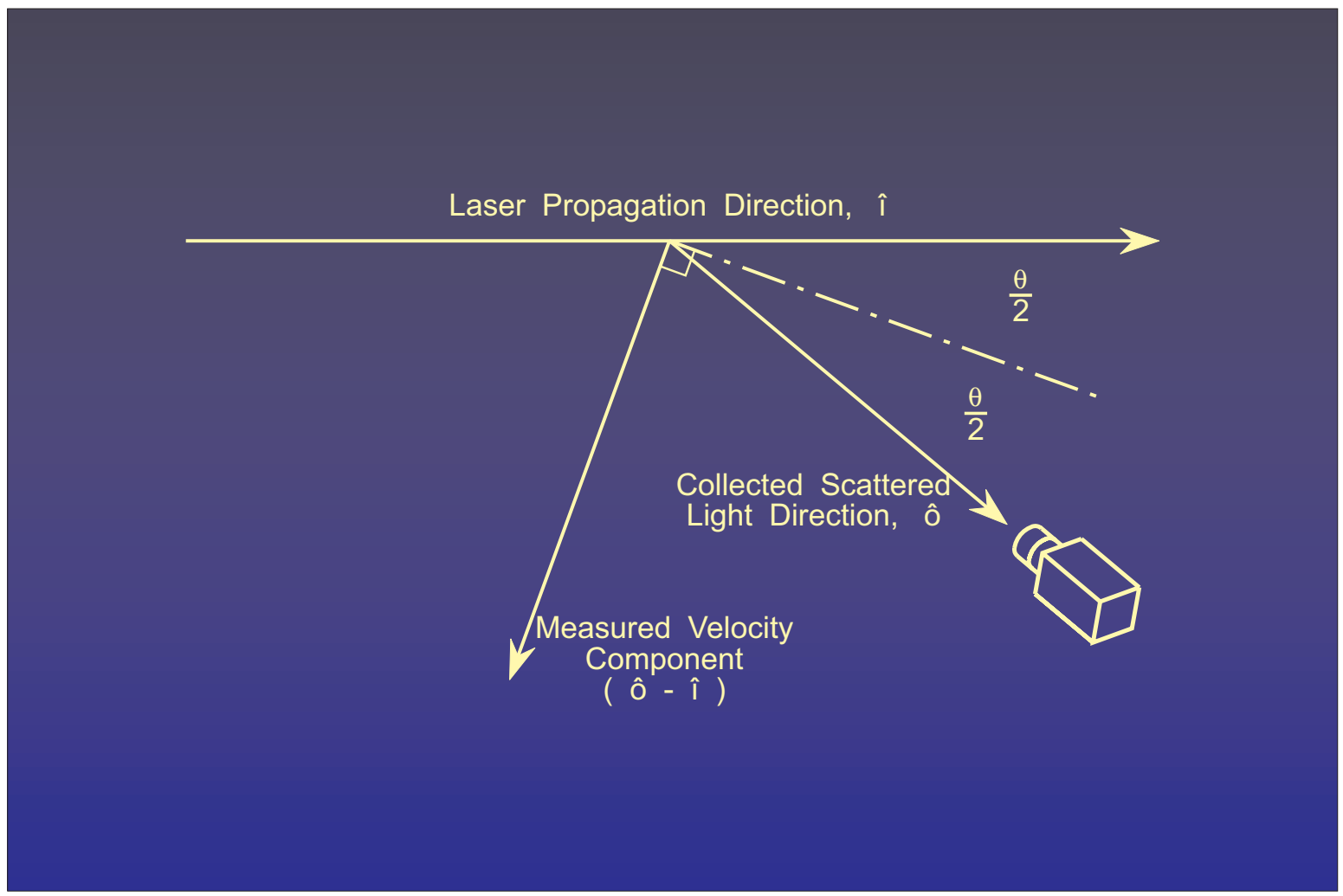

Figure 1.- Diagram depicting the velocity measurement direction based on the orientation of the laser propagation direction and the detector location. 


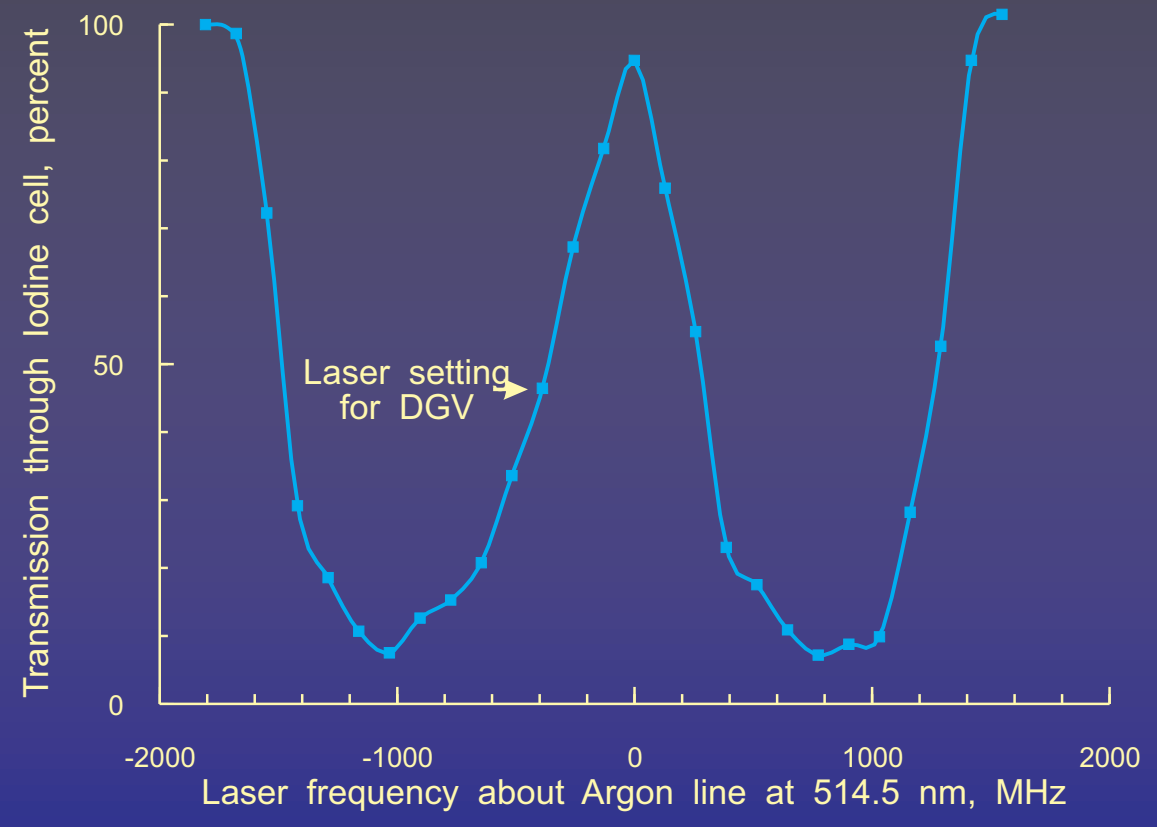

Figure 2.- Transfer function of the Iodine absorption line filter, ALF.

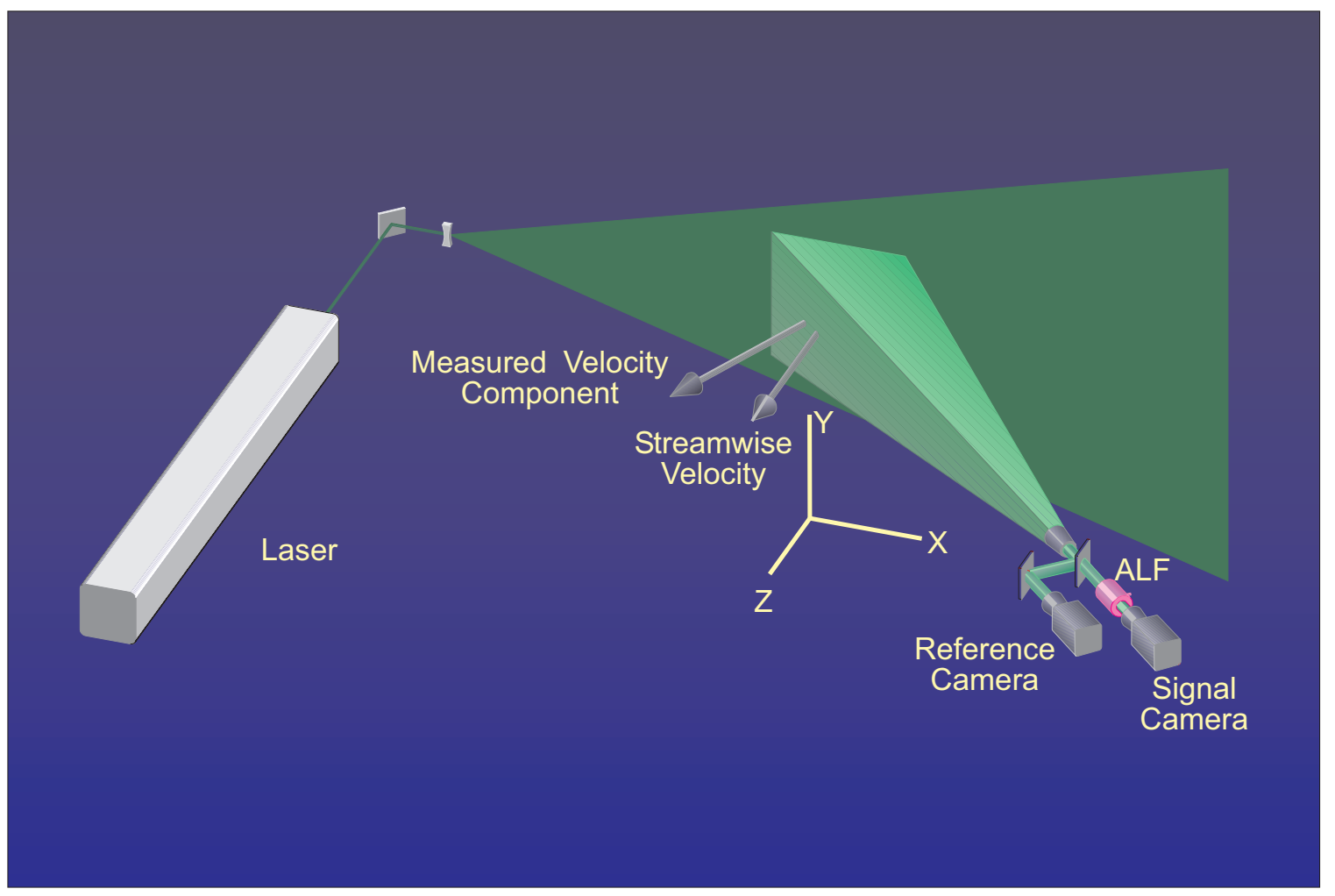

Figure 3.- Pictorial view of the Doppler global velocimeter used in the Basic Aerodynamics Research Tunnel. 


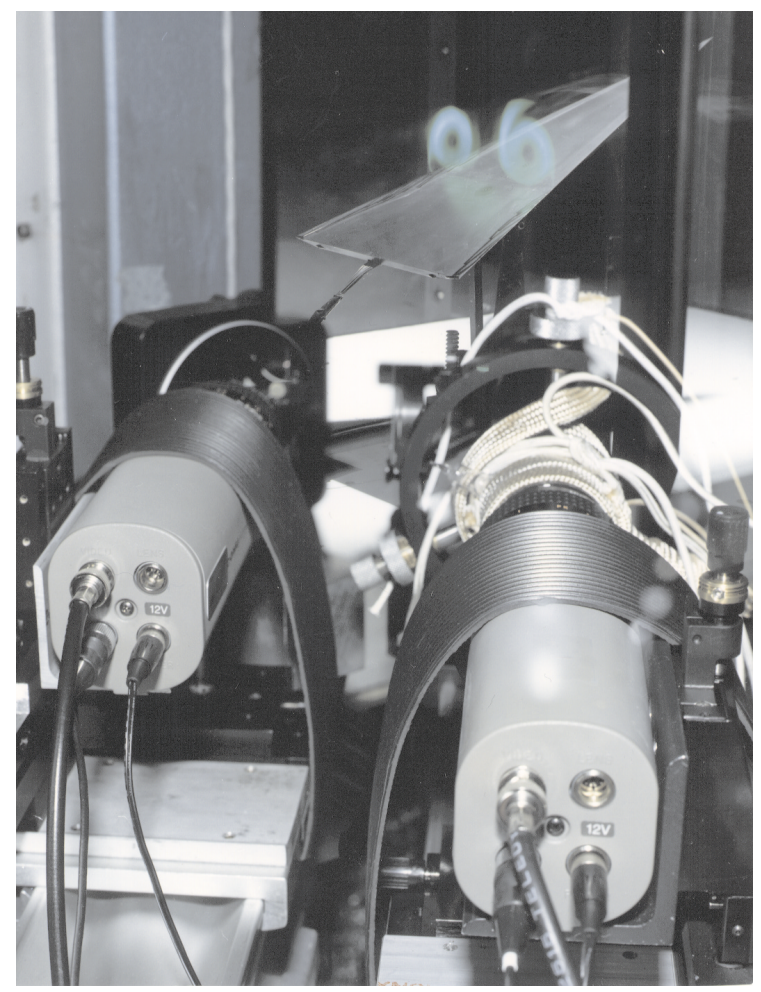

Figure 4.- Photograph of the Doppler global velocimeter installed in the Basic Aerodynamics Research Tunnel.

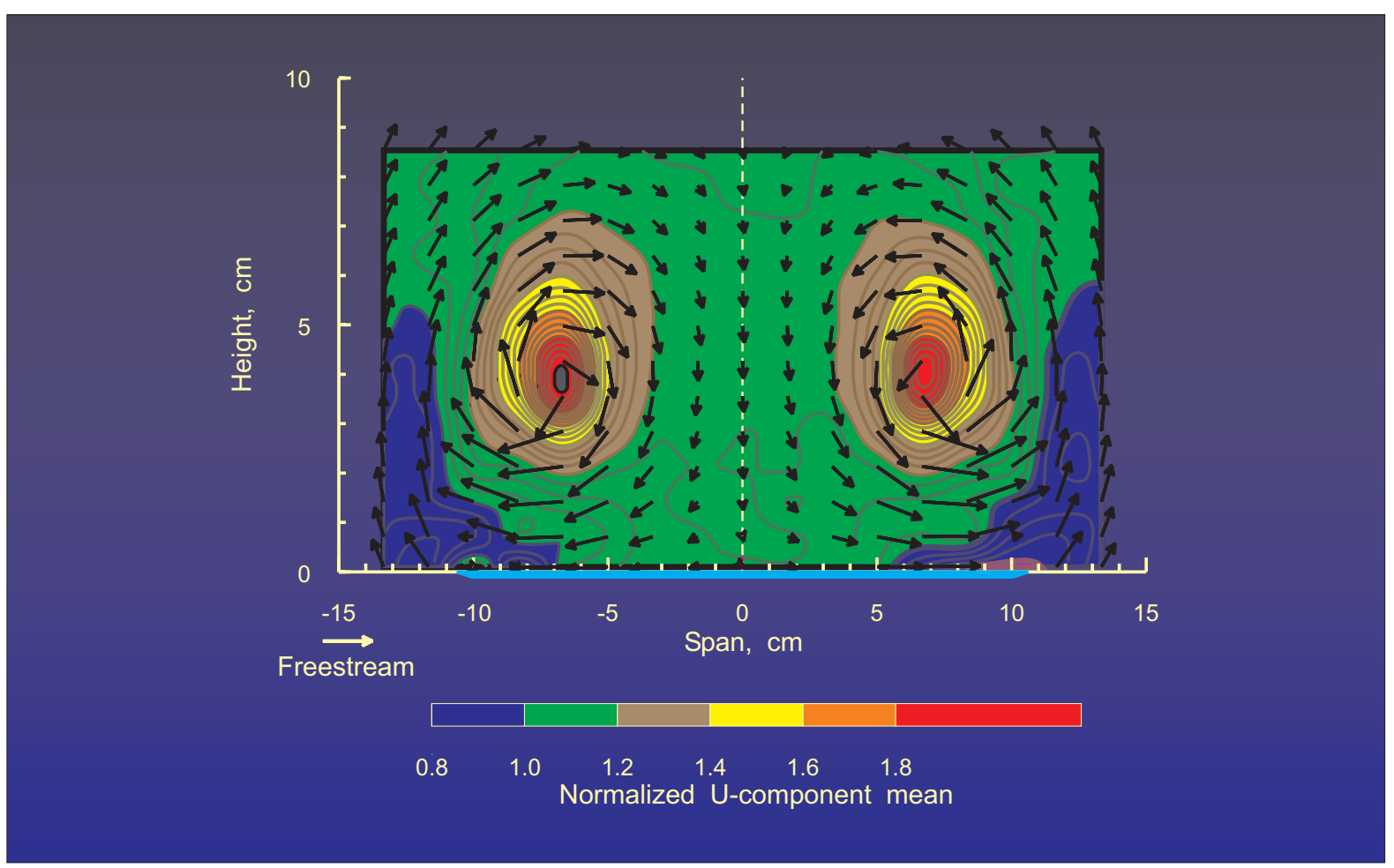

Figure 5.- Three component laser velocimeter measurements of the vortical flow field above a 75-degree delta wing at an angle-of-attack of 20.5 degrees. 


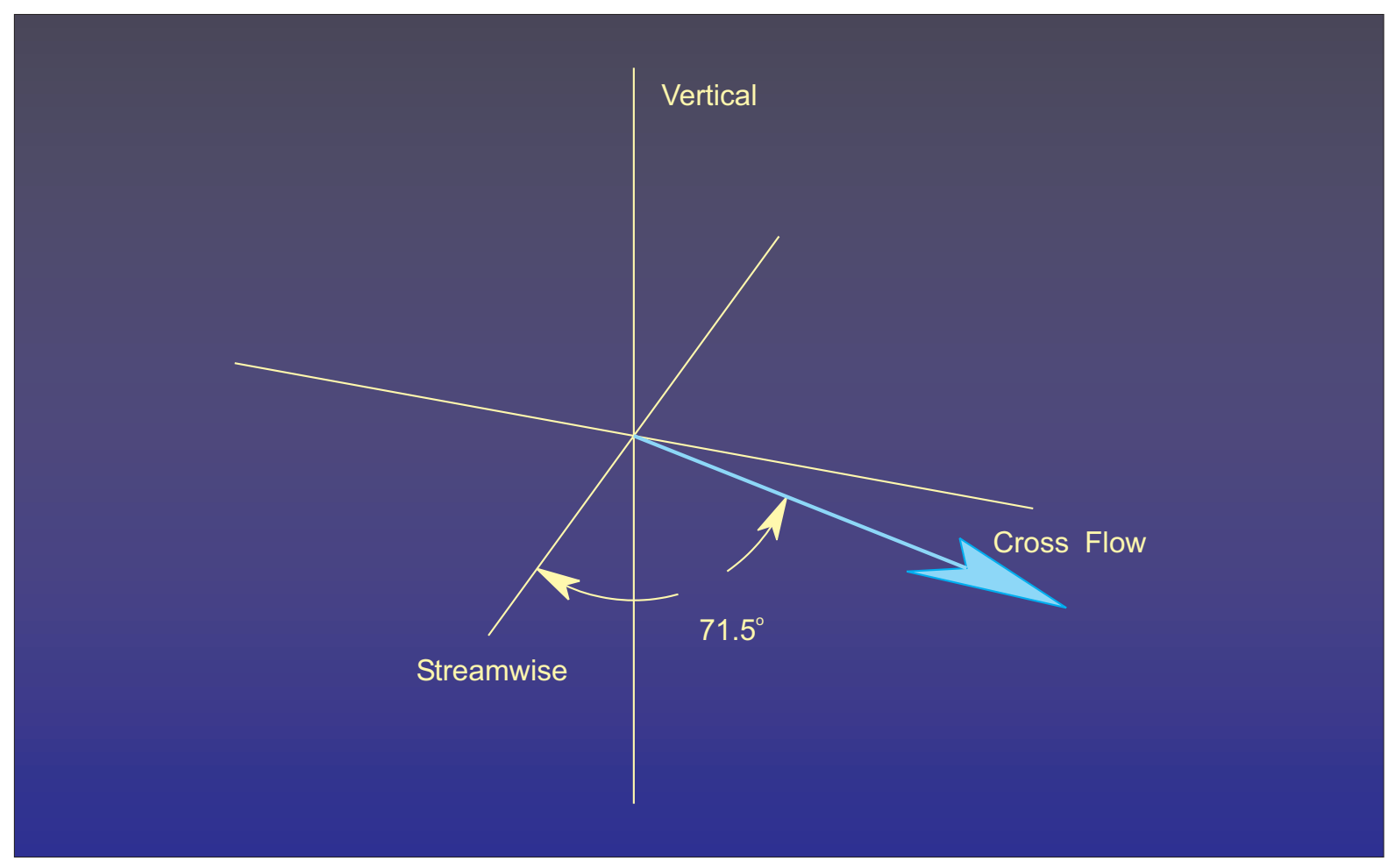

Figure 6.- Measurement direction for DGV operation in backscatter mode.

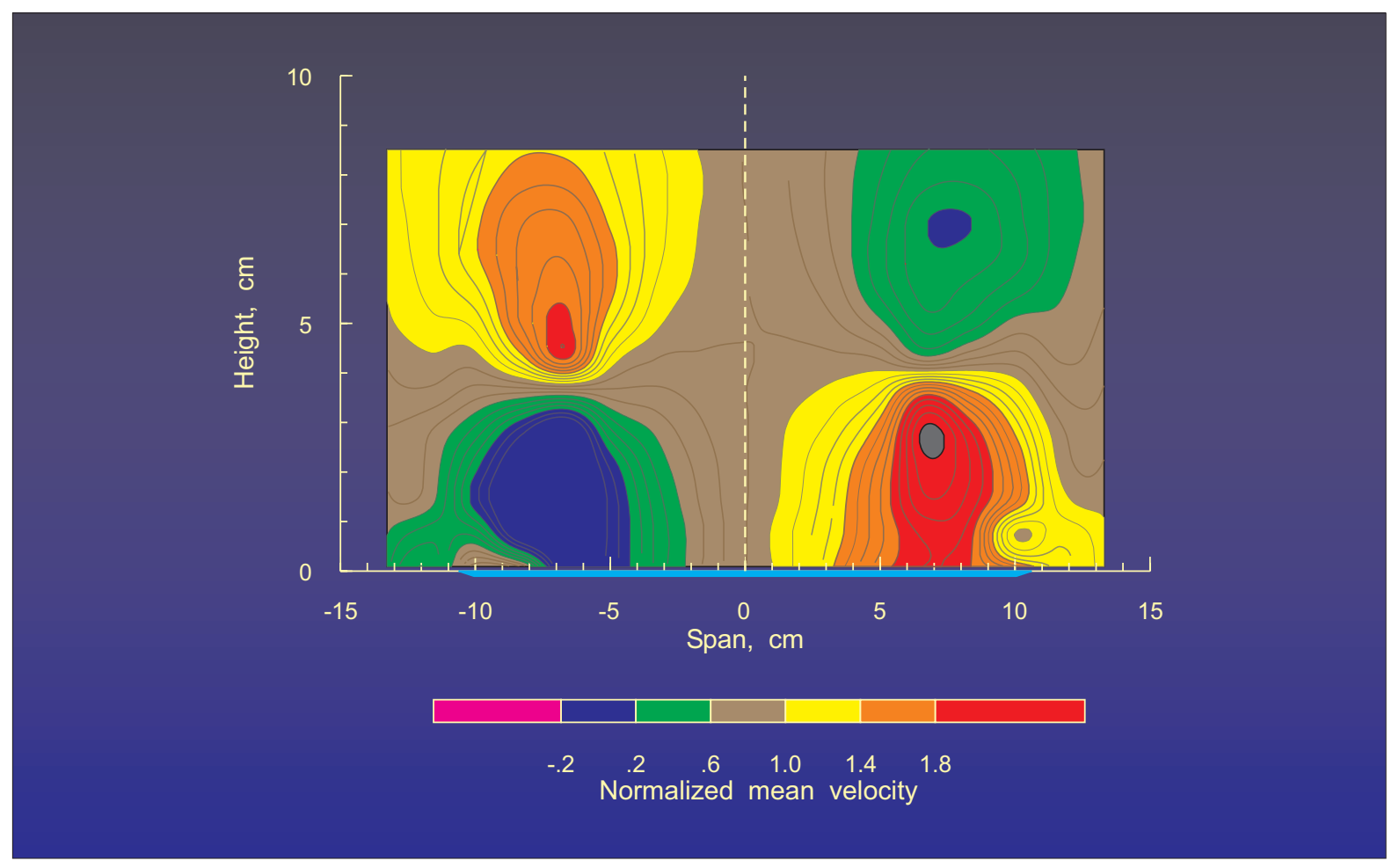

Figure 7.- Resolved laser velocimeter measurements along the direction 71.5 degrees from streamwise in the horizontal plane. 


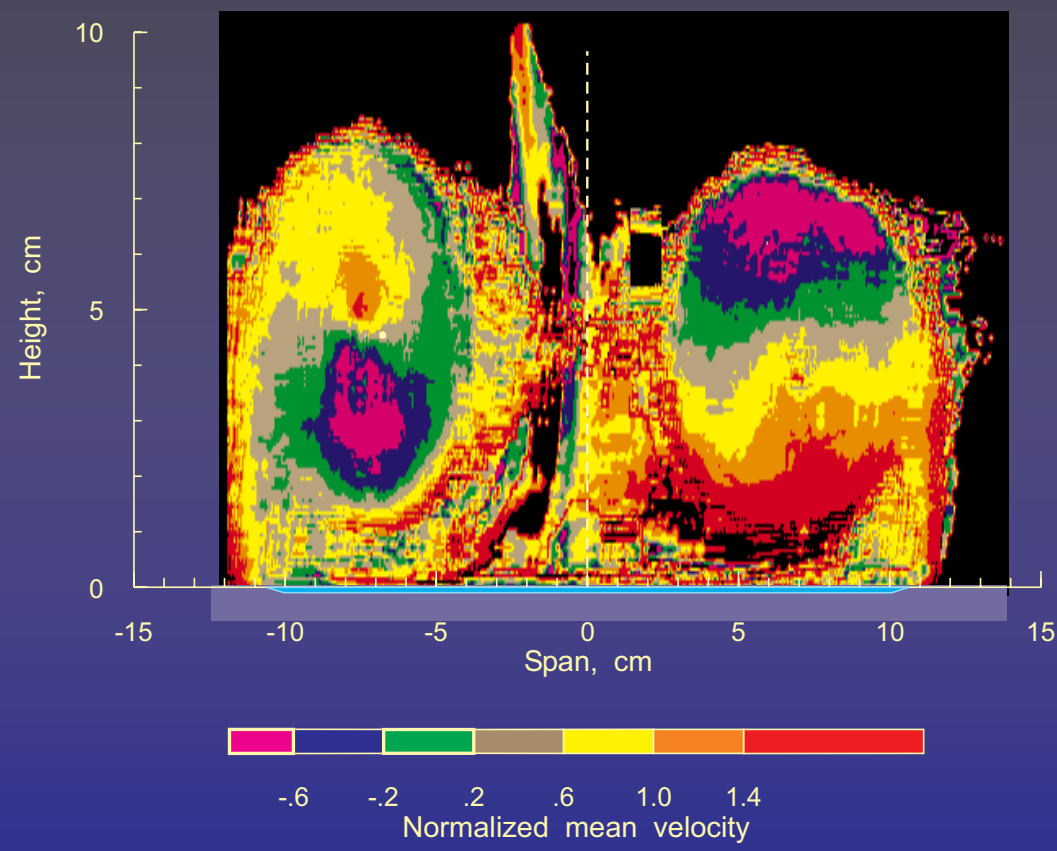

Figure 8.- DGV measurements of the velocity field (average of 30 frames) for the component along the direction 71.5 degrees from streamwise in the horizontal plane.

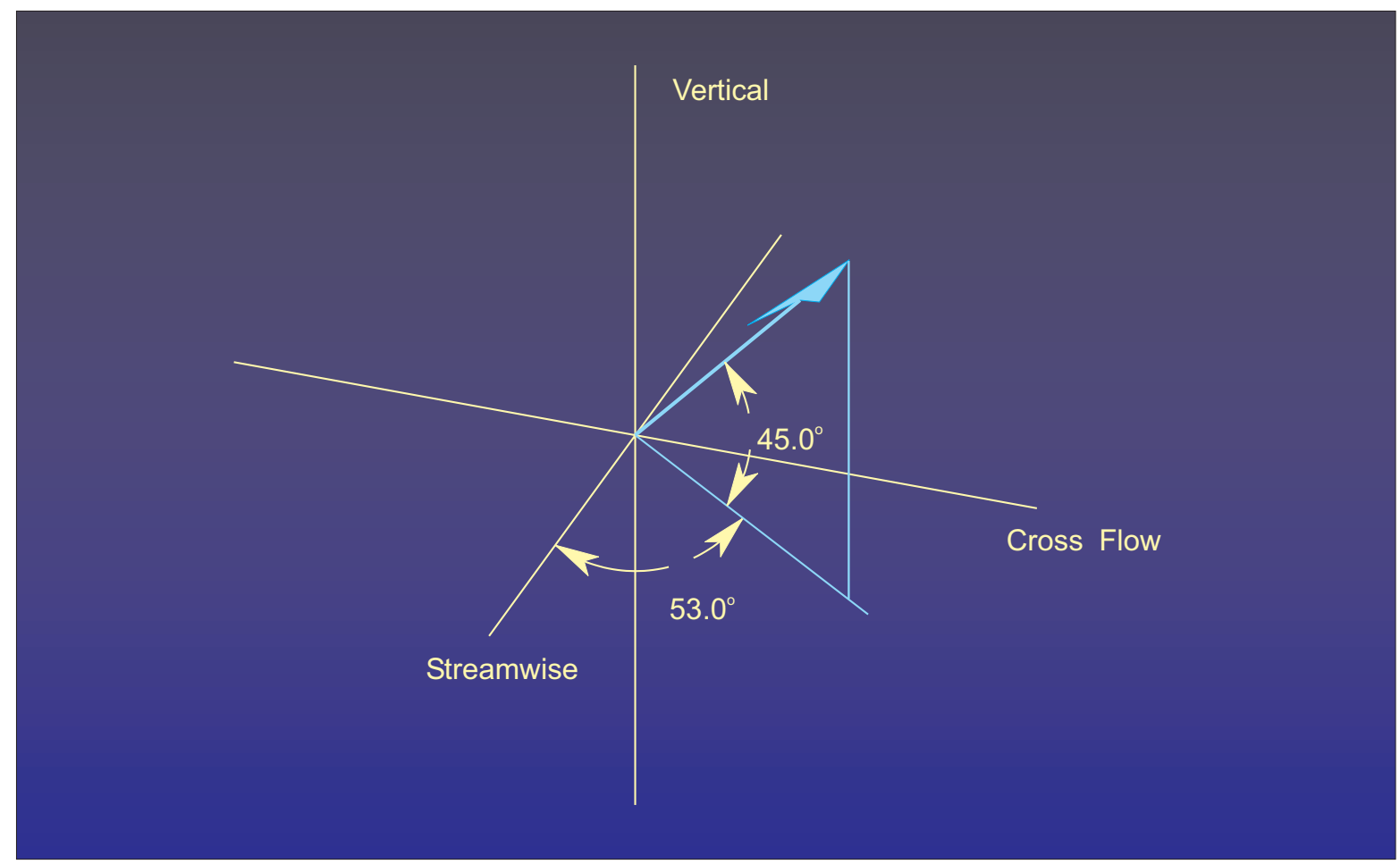

Figure 9.- Measurement direction for DGV operation in side scatter mode, (laser propagation from above). 


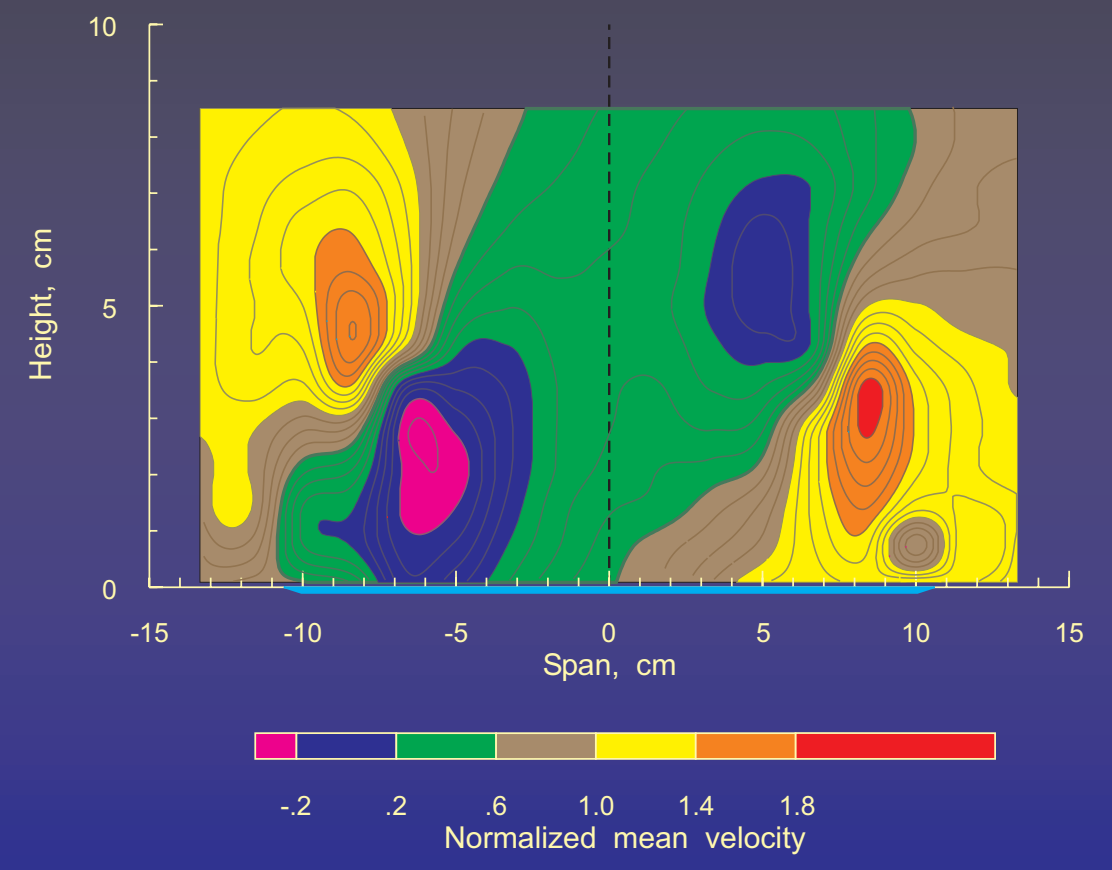

Figure 10.- Resolved laser velocimeter measurements along the direction 53.0 degrees from streamwise and 45.0 degrees above the horizontal plane.

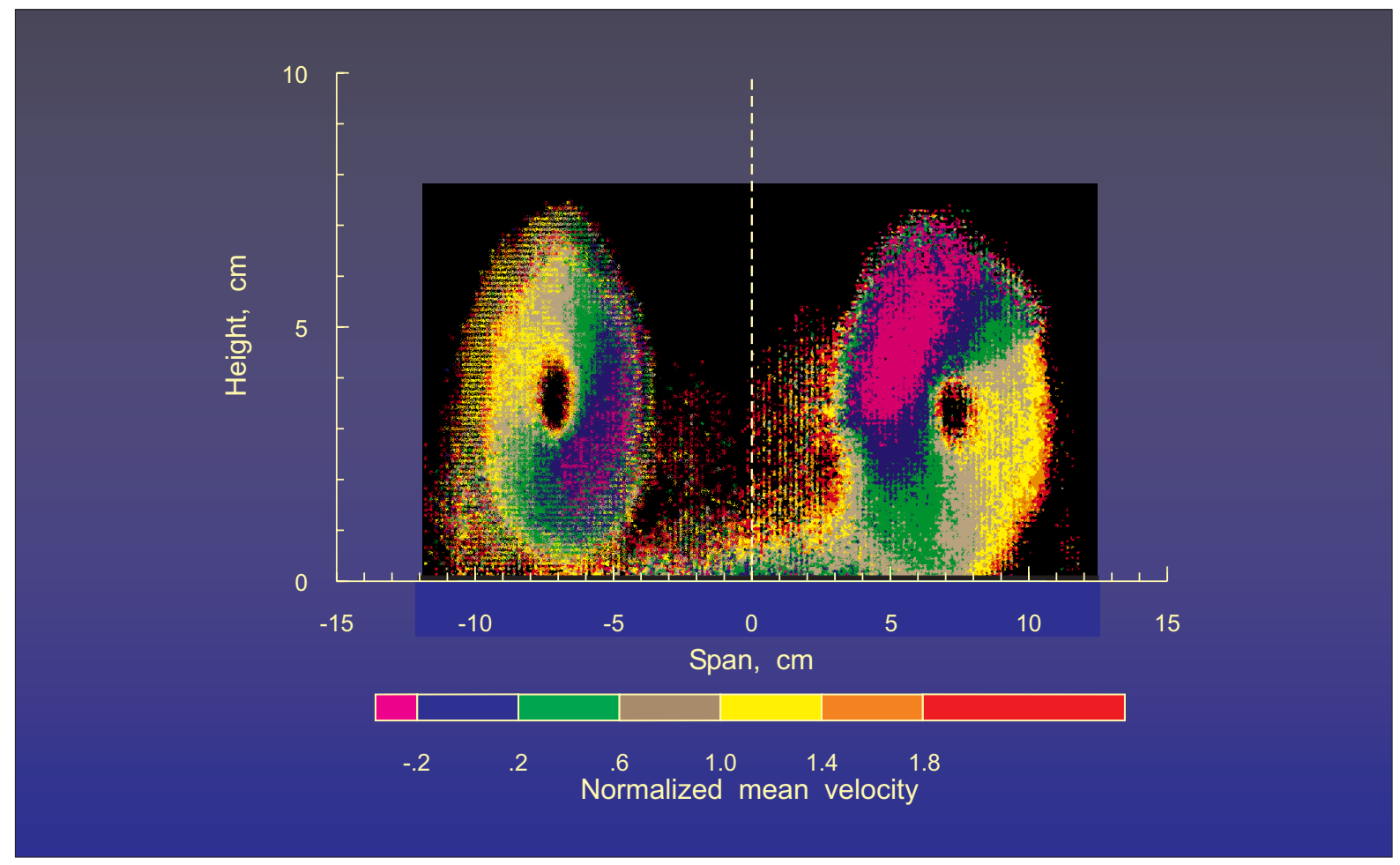

Figure 11.- DGV measurements of the velocity field (average of 30 frames) for the component along the direction 53.0 degrees from streamwise and 45.0 degrees above the horizontal plane. 


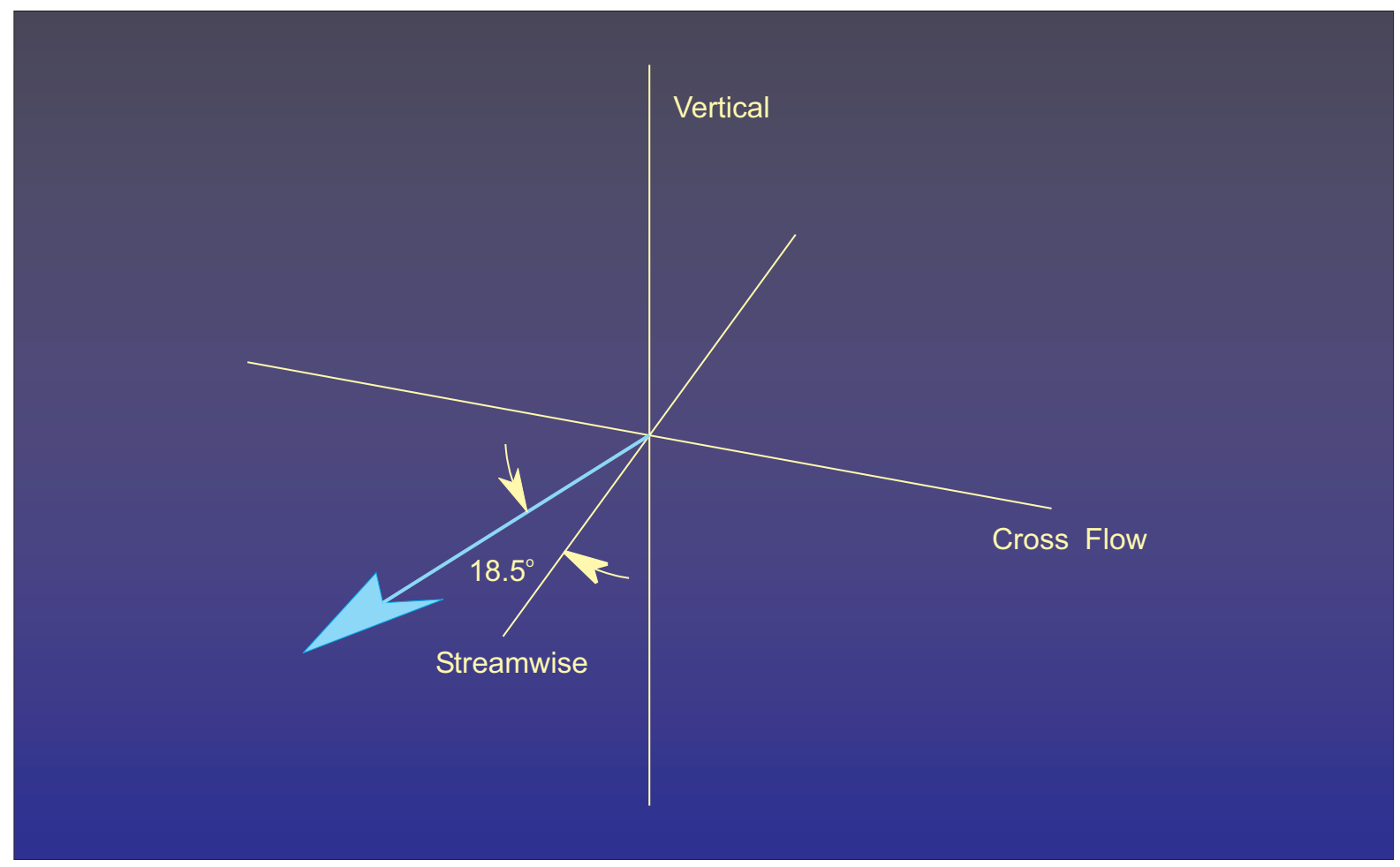

Figure 12.- Measurement direction for DGV operation in forward scatter mode.

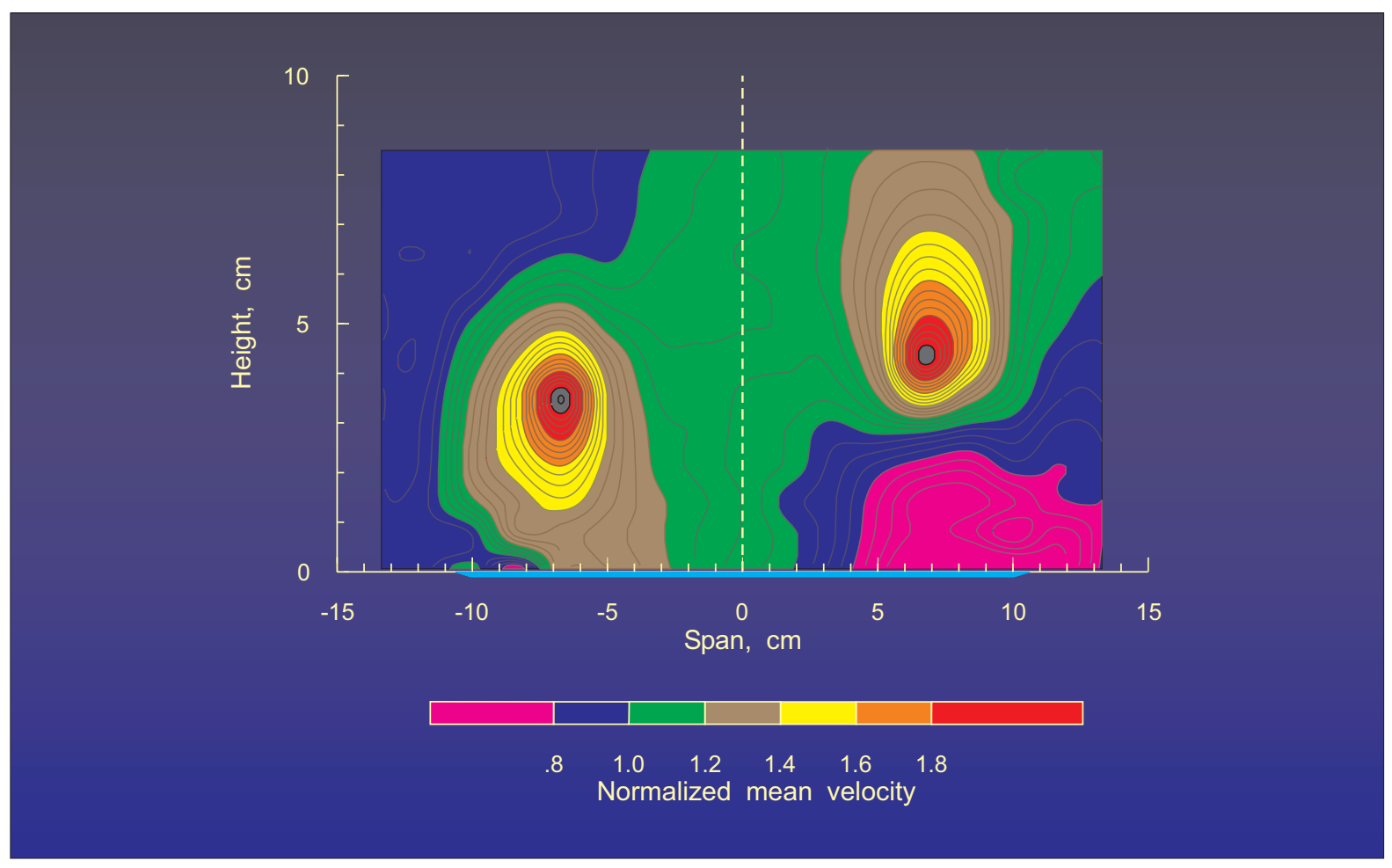

Figure 13.- Resolved laser velocimeter measurements along the direction 18.5 degrees from streamwise in the horizontal plane. 


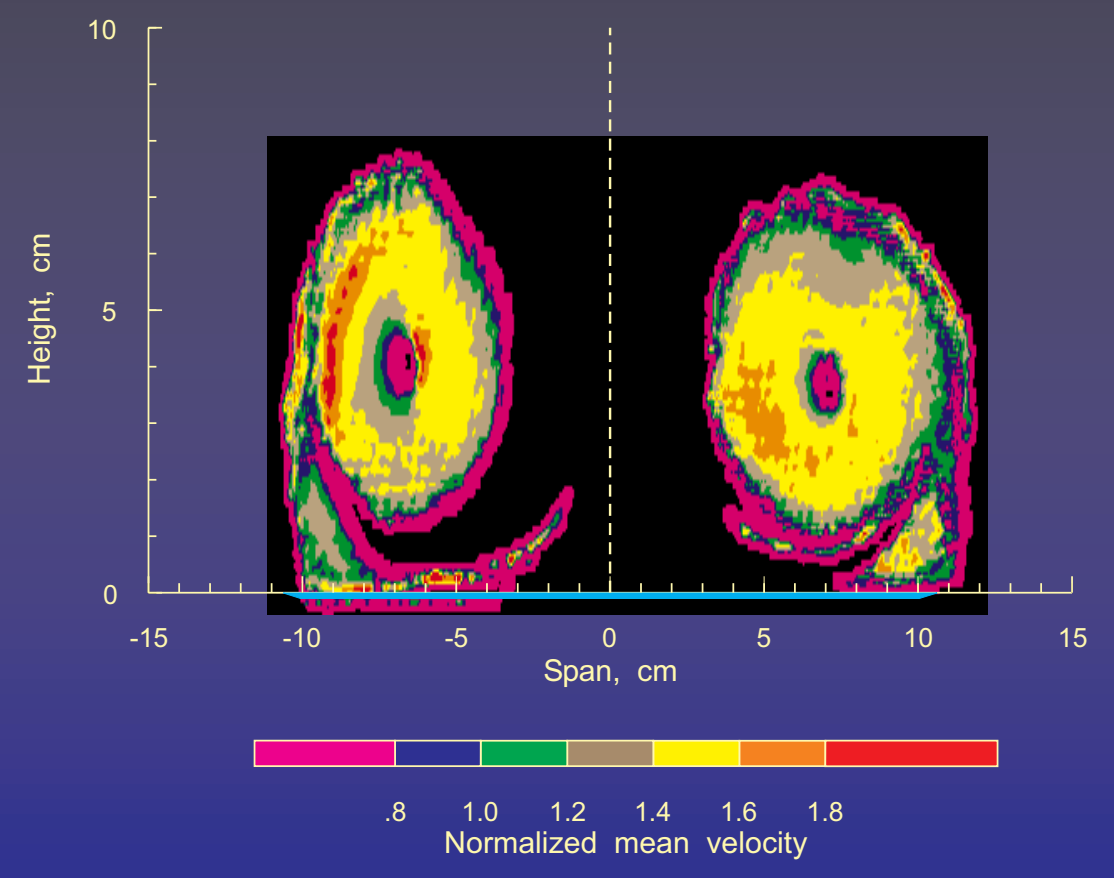

Figure 14.- DGV measurements of the velocity field (average of 30 frames) for the component along the direction 18.5 degrees from streamwise in the horizontal plane.
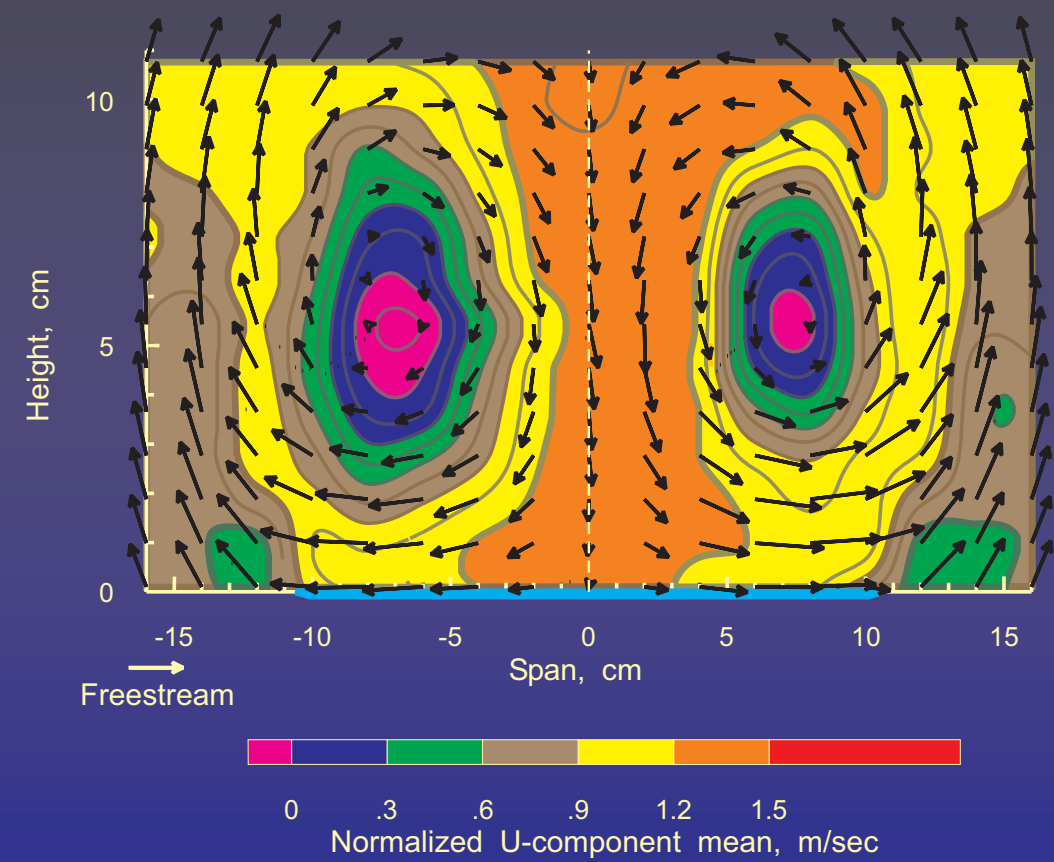

Figure 15.- Three component laser velocimeter measurements of the vortical flow field above a 75 -degree delta wing at an angle-of-attack of 40.0 degrees. 


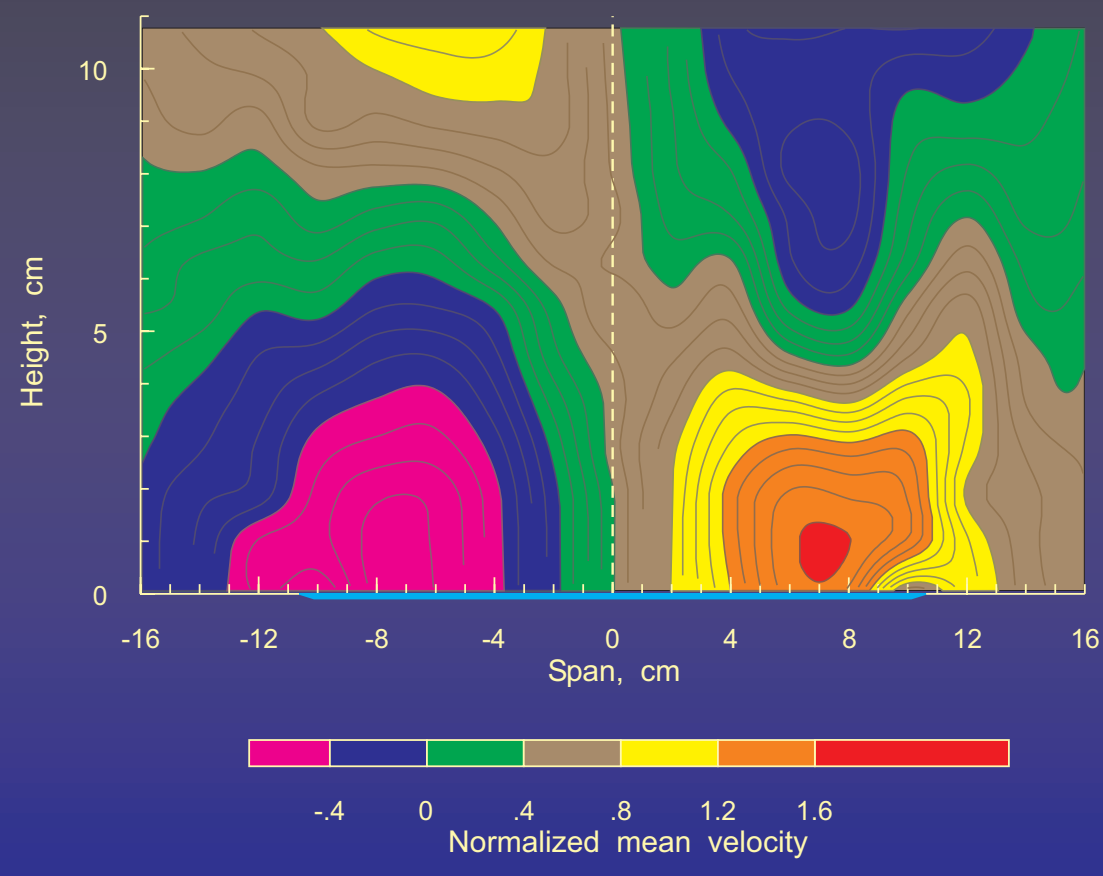

Figure 16.- Resolved laser velocimeter measurements along the direction 71.5 degrees from streamwise in the horizontal plane.

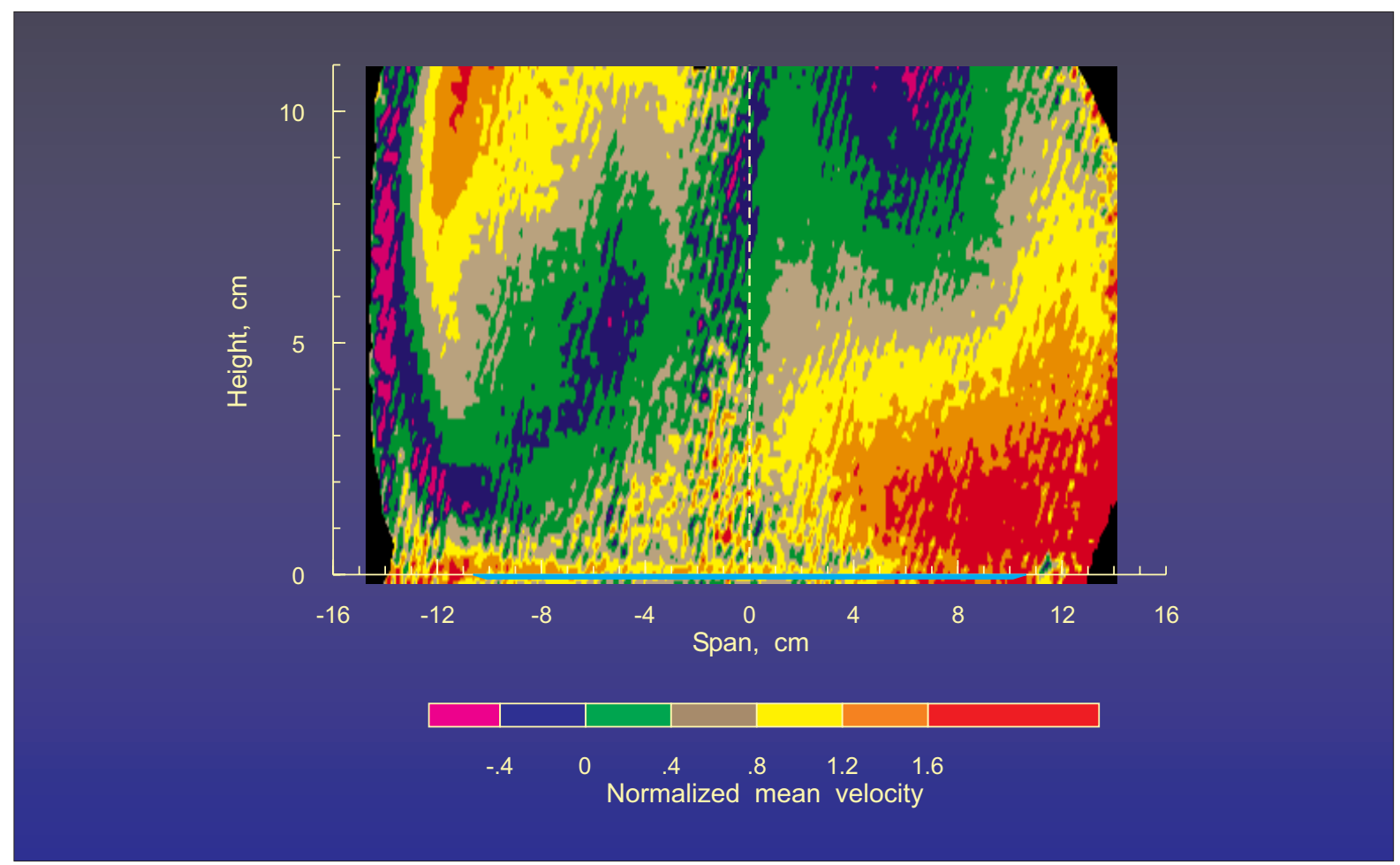

Figure 17.- DGV measurements of the velocity field (average of 30 frames) for the component along the direction 71.5 degrees from streamwise in the horizontal plane. 


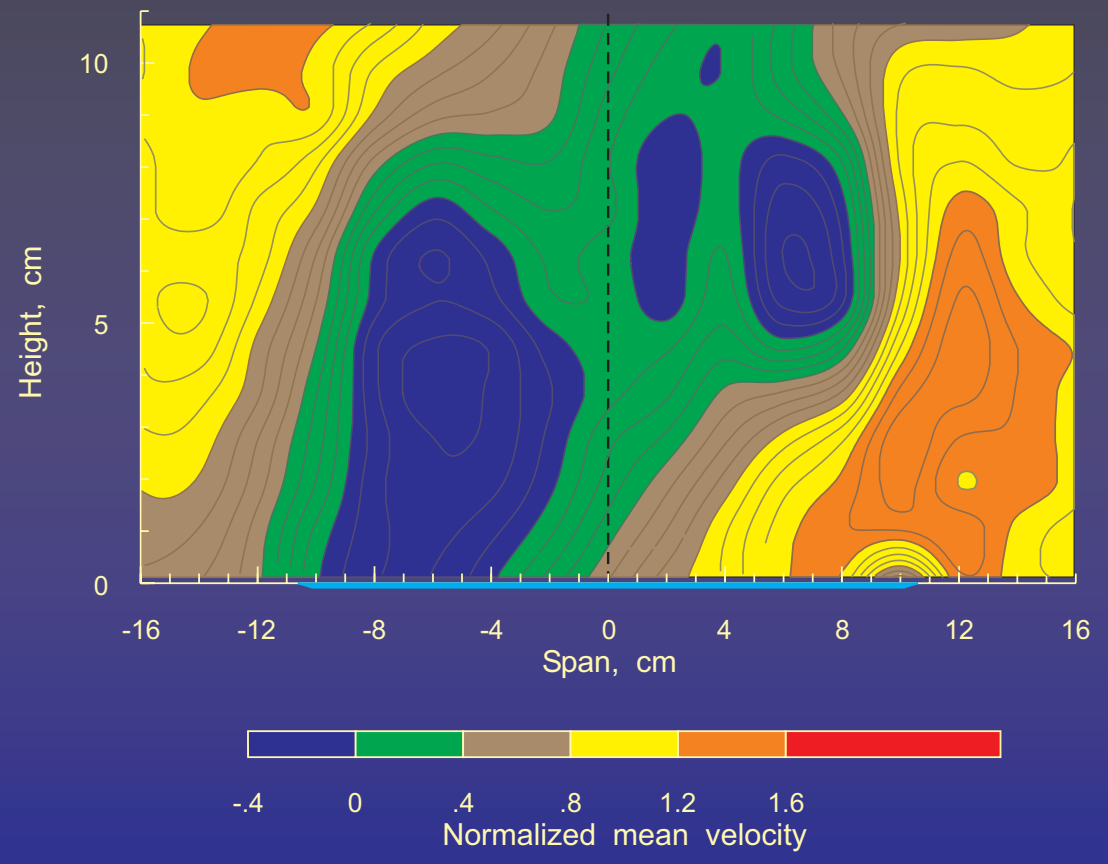

Figure 18.- Resolved laser velocimeter measurements along the direction 53.0 degrees from streamwise and 45.0 degrees above the horizontal plane.

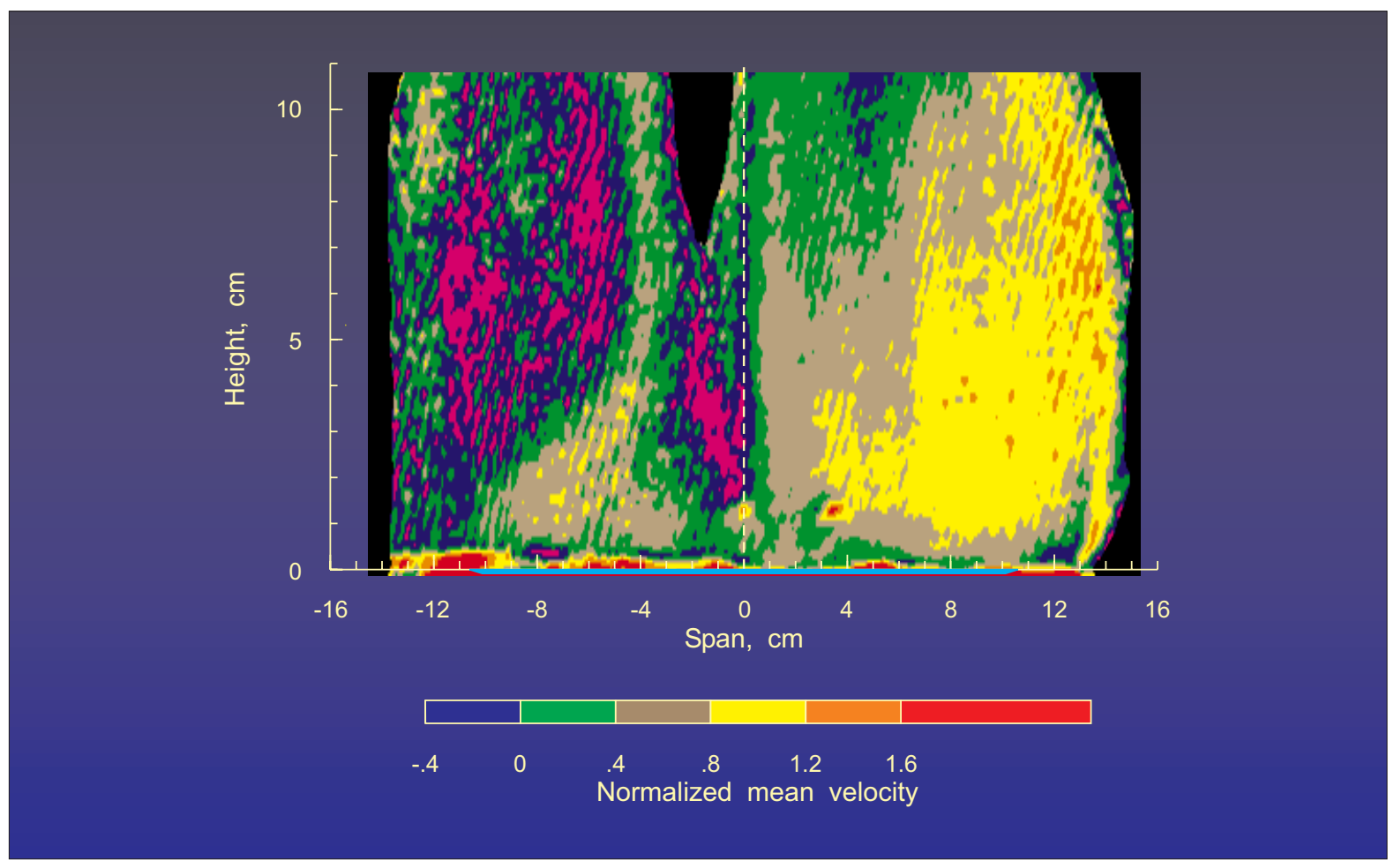

Figure 19.- DGV measurements of the velocity field (average of 30 frames) for the component along the direction 53.0 degrees from streamwise and 45.0 degrees above the horizontal plane. 


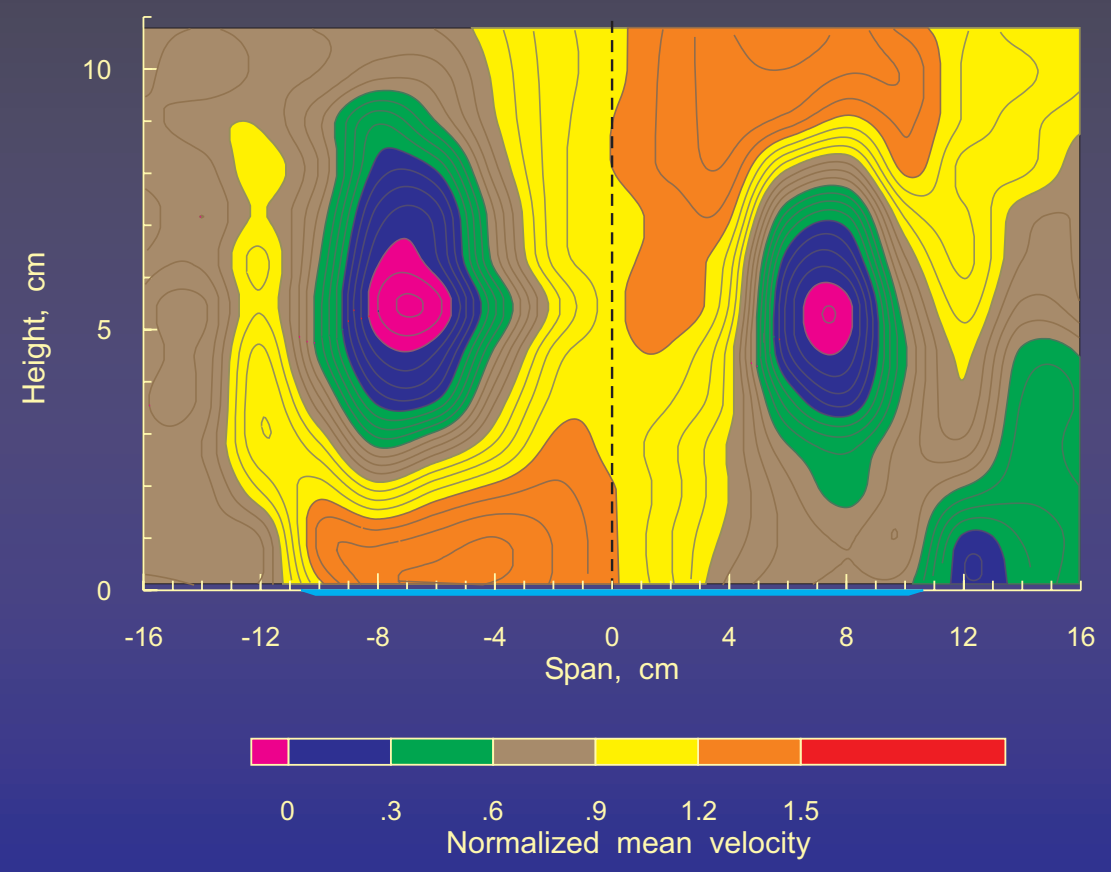

Figure 20.- Resolved laser velocimeter measurements along the direction 18.5 degrees from streamwise in the horizontal plane.

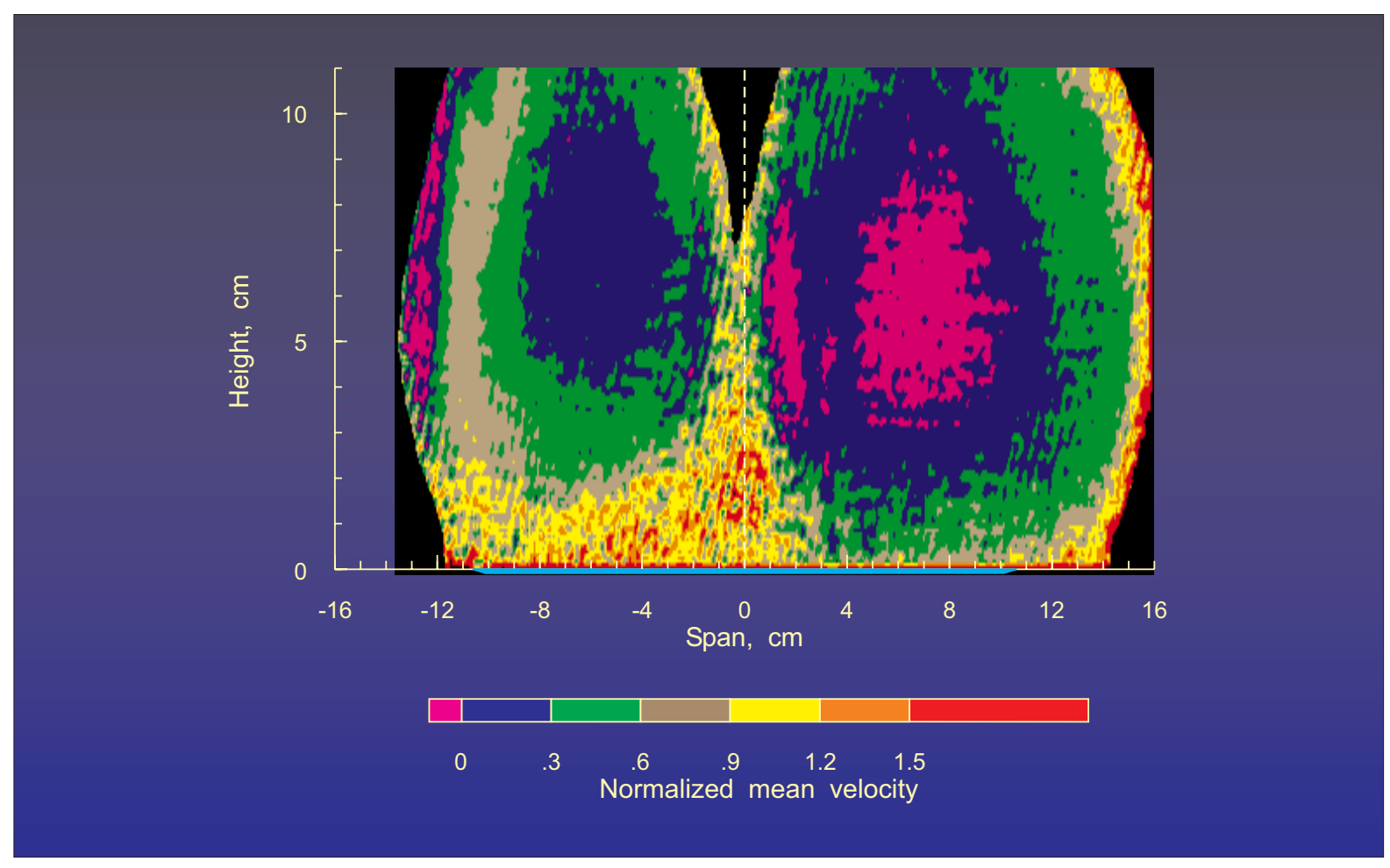

Figure 21.- DGV measurements of the velocity field (average of 30 frames) for the component along the direction 18.5 degrees from streamwise in the horizontal plane. 


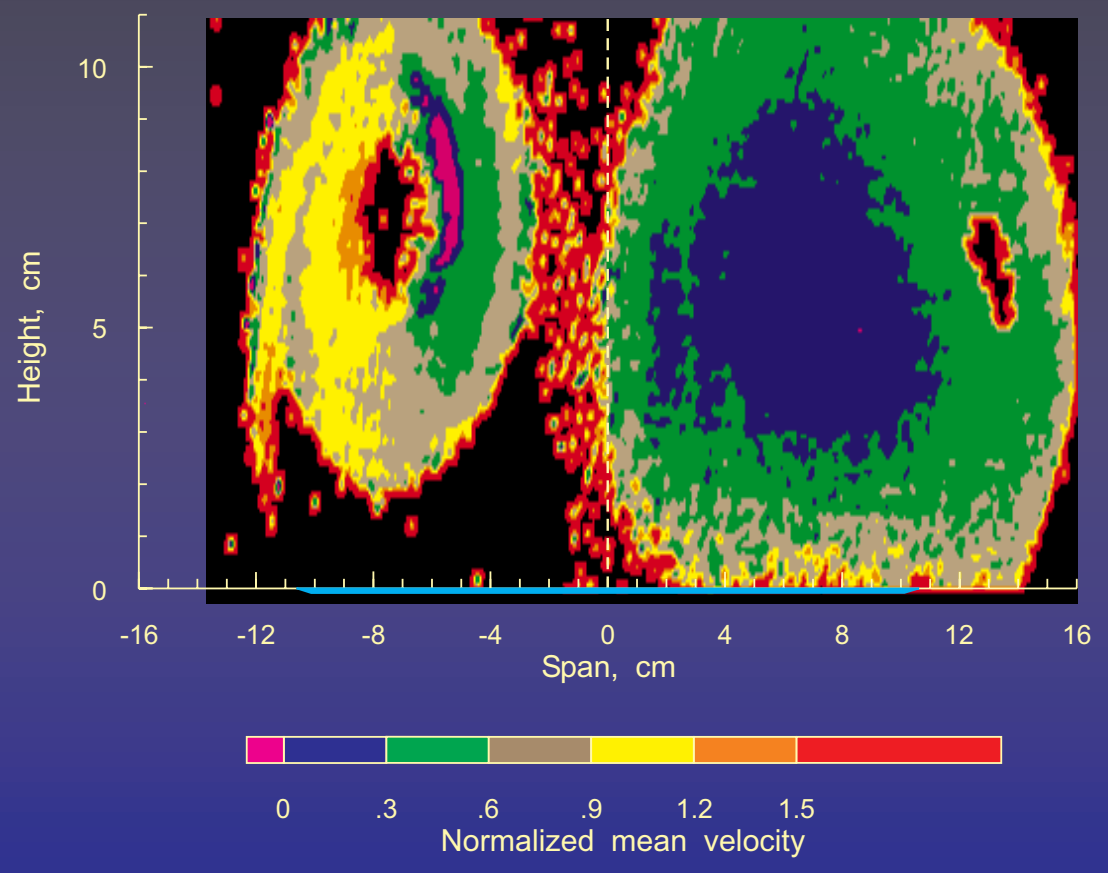

Figure 22.- DGV measurements of the velocity field (single frame) for the component along the direction 18.5 degrees from streamwise in the horizontal plane. 